\title{
Psychological Treatments in Functional Gastrointestinal Disorders: A Primer for the Gastroenterologist
}

\author{
Olafur S. Palsson, PsyD and William E. Whitehead, PhD \\ Center for Functional Gastrointestinal and Motility Disorders, and, Division of Gastroenterology \\ and Hepatology, University of North Carolina at Chapel Hill
}

\begin{abstract}
The functional gastrointestinal disorders (FGIDs) often show inadequate response to usual medical care. Psychological treatments can help improve FGID patient outcomes, and such treatment should be considered for patients who have moderate or severe symptoms after 3 to 6 months of medical care, and those whose symptoms are clearly exacerbated by stress or emotional symptoms. Effective psychological treatments, based on multiple randomized controlled trials, include cognitive behavioral therapy (CBT) and hypnosis for irritable bowel syndrome and pediatric functional abdominal pain; CBT for functional chest pain; and biofeedback for dyssynergic constipation in adults. Successful referral by the gastroenterologist for psychological treatment is facilitated by educating the patient about the rationale for such treatment, reassurance about the diagnosis and continuation of medical care, firm doctor-patient therapeutic alliance, and identification of, and communication with, an appropriate psychological services provider.
\end{abstract}

The functional gastrointestinal disorders (FGIDs) are a group of more than twenty chronic medical conditions of the GI tract that constitute a large proportion of the presenting problems seen in clinical gastroenterology and are hard to treat effectively. For example, in a survey of 1,658 patients with FGIDs in a Health Maintenance Organization in Seattle ${ }^{1}$ the proportion of patients who reported that their bowel symptoms were at least somewhat better after 6 months of usual medical management was only $49 \%$ for IBS, $63 \%$ for functional diarrhea, and $56 \%$ for functional constipation and functional abdominal pain. There is a clear need for supplemental interventions that can help reduce the morbidity, life impairment and chronically high healthcare utilization of the many FGID patients who remain highly symptomatic in spite of all that usual medical care approaches can offer. Psychological treatments have shown the best overall promise for that purpose to date, and are gradually becoming widely accepted and recommended options for FGIDs. For example, psychological treatments are given a "strong recommendation" rating for improving global IBS morbidity in the current evidence-based position statement of the American College of Gastroenterology ${ }^{2}$. Similarly, the AGA technical review on IBS recommends psychological treatment for moderate and severe patients, those with inadequate response to standard medical care and patients where psychosocial factors clearly exacerbate symptoms ${ }^{3}$.

(C) 2012 The American Gastroenterological Association. Published by Elsevier Inc. All rights reserved.

Corresponding Author: William E. Whitehead, PhD, Campus Box 7080, University of North Carolina at Chapel Hill, Chapel Hill, NC 27599-7080, Phone: (919) 966-6708, William_Whitehead@med.unc.edu.

Publisher's Disclaimer: This is a PDF file of an unedited manuscript that has been accepted for publication. As a service to our customers we are providing this early version of the manuscript. The manuscript will undergo copyediting, typesetting, and review of the resulting proof before it is published in its final citable form. Please note that during the production process errors may be discovered which could affect the content, and all legal disclaimers that apply to the journal pertain. 
The rationale for using psychological interventions for FGIDs can be summarized as follows:

1. Stressful life events trigger exacerbations of symptoms in many patients ${ }^{4}$ and traumatic life events such as sexual or physical abuse ${ }^{5}$ are associated with an increased prevalence of IBS and other FGIDs.

2. Co-morbid psychiatric disorders such as generalized anxiety disorder and major depression are highly prevalent in FGID patients (for example, found in 50-94\% of clinical samples of IBS patients ${ }^{6}$ ) and anxiety and depression have been identified as associated with poorer outcomes for FGID patients. ${ }^{7-9}$

3. The brain exerts a powerful influence over gastrointestinal pain perception, motility and secretion. In functional dyspepsia, anxiety is correlated with lowered threshold for gastric discomfort/pain and reduced gastric accommodation ${ }^{10}$, and depression is associated with increased postprandial distress, nausea, and vomiting ${ }^{11}$. In IBS patients, stress lowers visceral pain thresholds and stimulates colonic and ileal motility $^{4,12}$.

4. Psychological treatments work: A large number of randomized controlled trials show that short courses of certain psychological interventions can markedly improve the symptoms of several FGIDs while simultaneously enhancing emotional well-being and quality of life and sometimes reducing healthcare needs as well.

The dilemma of the clinical gastroenterologist is that he or she may be convinced that psychological treatment could help FGID patients but may not know which of the many forms of such therapies is suitable for a given disorder or how to go about making the referral. The aims of this article are to make this process easier by (1) identifying and describing the forms of psychological treatment that show evidence of effectiveness in FGIDs, (2) summarizing the empirical evidence for their effectiveness, (3) explaining how to find a suitable local provider, (4) characterizing which FGID patients should be considered for referral, and (5) describing how to make an effective referral.

\section{Empirically tested psychological treatments for FGIDs}

A number of different psychological therapies have been tested for FGIDs in the past 30 years. However, only five modes of treatment have been assessed in multiple randomized controlled trials (RCTs). That is a necessary standard of evidence for any firm conclusions to be made about the value of interventions for FGIDs for multiple reasons: Without randomization, selection bias is likely to confound treatment outcomes; placebo rates are often high for psychological interventions so placebo control or credible active treatments are necessary for outcome comparisons; and psychological treatments are generally carried out within the context of continued medical care, so observed therapeutic responses cannot be conclusively attributed to psychological treatment without control groups.

Although numerous systematic reviews have been published in the past on psychological treatments for various FGIDs, these are outdated. Some of the most important and strongest trials have appeared in the literature only after key reviews were published. We therefore searched the research literature via Medline (1965-2012) for all RCTs on psychological treatments for FGIDs (using terms for various common psychological treatments and the individual FGIDs), and reviewed papers cited in past systematic reviews as well as the reference sections of the papers found in our online search. Five psychological therapies cognitive behavior therapy, hypnosis, psychodynamic interpersonal therapy, relaxation training, and biofeedback - have been tested in multiple RCTs (see supplemental tables 15), and our review will be limited to those five therapies. As gastroenterologists may not 
have detailed knowledge of the nature of those therapies, we will describe each of them and then summarize the evidence for their value in FGID treatment. We will exclude from this overview several studies that combined multiple different psychological treatments sometimes as many as four or more different interventions applied simultaneously -- as this makes it impossible to judge the value of a particular therapy approach.

\section{Cognitive Behavioral Therapy (CBT)}

This is a structured form of psychotherapy that is usually conducted individually but can be administered in group format. The treatment usually consists of a course of 6-12 sessions that focus on the present situations in which symptoms occur rather than the patient's past history. CBT is based on the theory that maladaptive thoughts are the causes of psychological symptoms such as anxiety and depression, which in turn cause or exacerbate physical symptoms. An example would be a patient who believes that eating in a public place will always cause them to have diarrhea and other embarrassing symptoms (a catastrophizing maladaptive thought), which might lead the patient to both avoid social interactions (self-defeating behavior) and to become anxious when dining in a restaurant. The anxiety and autonomic arousal caused by this maladaptive thought may actually trigger diarrhea. The therapist aims to help the patient recognize maladaptive thoughts and selfdefeating behavior patterns that are adversely affecting life functioning, symptom experience and mental well-being. Therapy tasks commonly include increasing awareness of the association between stressors, thoughts, and symptoms; examining and correcting irrational beliefs; countering automatic negative thoughts; observing and problem-solving factors that exacerbate symptoms; and identifying and adopting alternative, more effective coping strategies to handle challenging life situations and deal with gastrointestinal symptoms. In between therapy visits, patients are typically asked to complete homework assignments related to the treatment tasks. It should be noted here that the relative emphasis on individual treatment components varies a lot. Some interventions that fall under the general umbrella of CBT are mostly or exclusively either cognitive or behavioral in nature: i.e., they either focus on changing thought patterns or on learning and practicing healthy behavior patterns.

CBT has been studied more than any other form of psychological intervention for FGIDs in randomized controlled trials. Thirty RCTs have been published (Supplemental Table 1) comparing CBT to other interventions. The majority of these trials (18 studies) were conducted on adults with IBS. Outcomes for CBT treatment were compared to control groups receiving usual medical care or on waiting lists for the treatment, antidepressant or antispasmodic medication, placebo or active psychological interventions such as supportive therapy, education, or stress management/stress reduction treatment. This substantial body of empirical studies shows that CBT is an effective therapy for improving IBS. In all but three trials, the CBT arms showed superior outcomes. In the positive trials, gastrointestinal symptoms were almost uniformly found to be significantly reduced after treatment, sometimes substantially more than in comparison groups. For example, Payne and Blanchard ${ }^{13}$ randomized 34 patients to either 8 weeks of cognitive therapy, a self-help support group (which controlled for "placebo" or expectancy effects), or a waiting list group. Cognitive therapy patients showed an average of $67 \%$ reduction in the composite bowel symptom score after treatment, compared to $31 \%$ reduction in the support group and only $10 \%$ in the waiting list subjects. Improvement was fully maintained at 3-month followup. While most studies have not included follow-up longer than 3 months post-treatment, there is evidence that therapeutic benefit of CBT for IBS can last 8 months to 2 years after treatment termination ${ }^{14-16}$. In addition to GI symptom improvement, quality of life and emotional well-being are often documented to improve significantly from such treatment as well. 
CBT has also proven to be a reliably effective therapy for the treatment of functional chest pain and recurrent abdominal pain in children (Supplemental Table 1), both of which are conditions for which no good pharmacologic therapy exists.

Five RCTs on non-cardiac chest pain all found superior outcomes for patients assigned to CBT compared to control groups, with four of them showing the treatment to be effective in reducing the pain problem ${ }^{17-20}$ and the fifth (which used the shortest course of treatment only 3 sessions) finding only an effect on quality of life ${ }^{21}$. Especially noteworthy is the study by Spinhoven and colleagues ${ }^{20}$, who assigned 69 patients to either CBT, paroxetine or usual medical care. The CBT patients had a greater reduction in pain than both the medication and usual care groups, and only the CBT group showed reduction in heartfocused anxiety after treatment (even though paroxetine is often used to treat anxiety problems). Six RCTs have tested CBT for recurrent abdominal pain in children. In such treatment, parents and children are commonly both treated together. Here again, all the trials have found CBT to lead to better outcomes (diminished pain), with CBT generally leading to longer term pain reduction and higher rates of elimination of pain compared to control groups.

A noteworthy recent development in the field of CBT for FGIDs is testing of mostly or entirely self-administered CBT, with minimal involvement of clinicians or staff. The internet, printed manuals, or PDAs serve as the principal mode of therapy delivery, and this addresses the important limitation of lack of CBT therapists in many geographical areas. Seven such randomized trials (identified with an asterisk in supplemental Table 1) have been reported so far, mostly in IBS treatment. All of them show that this method of delivering CBT leads to superior outcomes compared to the control conditions. Moreover, the largest randomized-controlled trial ever conducted on psychological treatment for functional gastrointestinal disorder, a multicenter NIH-funded trial with estimated enrollment of 480 IBS subjects ${ }^{22}$, is currently evaluating the potential of self-administered CBT further. Even though such cost-effective therapy is not yet generally available, the consistently positive findings to date suggest that a very inexpensive and easily accessible form of effective CBT may be on the horizon for IBS and perhaps other FGIDs.

\section{Hypnosis treatment}

Clinical hypnosis is a verbal intervention that utilizes a special mental state of enhanced receptivity to suggestion to facilitate therapeutic psychological and physiological changes. Treatment sessions, which are generally conducted one-on-one, begin with an induction of the hypnotic state. This is accomplished in various ways that generally involve relaxation, narrowing and intensification of the focus of attention (for example, by means of eye fixation), and the patients' gradual release of deliberate control of their mental activity. Once the hypnotic state has been achieved, deepening of the altered state generally follows with the aid of counting, physical relaxation and guided mental dissociation from the here-andnow. The hypnotherapist then conducts the clinical intervention, which is composed of targeted verbal suggestions and therapeutic imagery to encourage improvement in symptoms. In the treatment of FGIDs, imagery and suggestions commonly aim at regulating smooth muscle activity, reducing the impact of stress on GI symptoms, reducing gut pain perception and attention to symptoms, and increasing the patient's sense of control over symptoms. Examples of imagery used in IBS hypnosis treatment are a mental image of the intestinal wall being coated with a strong protective coating that makes it immune to irritation or pain, or imagining the bowel as a river and the patient mentally slowing or speeding the flow of the river to counter diarrhea or constipation. Similar to CBT, hypnosis treatment for FGIDs usually consists of a course of 6-12 therapy sessions, and patients are 
commonly asked to practice hypnosis at home in between visits with the aid of audio recordings.

Hypnosis treatment has been tested for FGDs in 11 published RCTs to date (Supplemental Table 2). Control groups have included supportive talk therapy, placebo pills, waiting lists, multi-component (non-hypnotic) audio recordings, and usual medical care. Seven of these trials were on IBS in adults, and all except one of those RCTs found hypnosis to result in significantly greater improvement in gastrointestinal symptoms than seen in the comparison groups. Emotional symptoms and quality of life also improved significantly in studies where these parameters were measured. Improvement from hypnosis treatment generally lasts a long time. Two of the RCTs reported therapeutic gains in treatment responders to be fully maintained at $10^{23}$ and $18^{24}$ months after treatment, respectively. Separately, one of the investigative teams published a large case series of 204 consecutive IBS patients whose symptoms were reassessed annually for years after hypnotherapy, and reported that $81 \%$ of patients maintained their treatment gains fully for at least 5 years ${ }^{25}$. Overall, the data strongly indicate that hypnosis treatment is an effective intervention for IBS.

Two controlled studies have investigated the impact of hypnosis treatment on pediatric abdominal pain, both with impressive results. Vlieger and colleagues in the Netherlands randomly assigned 53 children with functional abdominal pain to either 6 sessions of hypnotherapy or supportive therapy ${ }^{26}$. The pain improved far more in the hypnosis group, and at 1 year follow-up, $85 \%$ of the hypnosis subjects and $25 \%$ of controls were treatment responders. At five-year follow-up, the pain of a significantly higher proportion of the hypnosis subjects than controls was in remission $(68 \% \text { vs. } 20 \%)^{27}$. In the second study, conducted in the U.S. by our research team ${ }^{28}$, children were randomized to either guided imagery treatment (a variant of hypnosis that used hypnotic suggestions and vivid imagery without formal hypnotic induction) or usual medical care. The hypnosis intervention was self-administered by the children at home via audio recordings. At the end of treatment, pain was reduced by half or more compared to pre-treatment assessment in $73.3 \%$ of the hypnosis group versus only $26.6 \%$ of the usual medical care group. Although it is desirable for more studies to be conducted on this application of hypnosis, this pair of trials indicates that hypnosis is a good option for reducing pediatric abdominal pain.

Finally, two initial randomized placebo-controlled investigations have found hypnosis treatment to lead to dramatically greater immediate and long-term improvement in the symptoms of non-cardiac chest pain ${ }^{29,30}$ and functional dyspepsia ${ }^{31}$ compared to supportive talk therapy, placebo pills, and - in the functional dyspepsia trial - compared to ranitidine as well. These FGIDs therefore appear to be promising new targets for hypnosis intervention, but further empirical work is needed to evaluate those applications of this therapy.

\section{Relaxation training}

This category of psychological treatment describes a heterogeneous group of interventions that principally aim to reduce sympathetic nervous system arousal and lessen physiological stress reactivity. Relaxation training is often included as a component of other interventions (such as CBT) and has also been used as control treatment for other psychological treatment. However, we identified 7 randomized studies where such treatment was tested as a monotherapy for FGDs, all of them in IBS (supplemental table 3). One sizable trial, by Boyce and colleagues, compared outcomes for relaxation training with those of cognitivetherapy and standard medical care and found no outcome differences between the treatment arms $^{32}$. In contrast, the other 6 RCT all found some significant benefits of relaxation training not seen in the comparison groups. Therefore it seems that interventions that aim at reducing autonomic arousal and stress reactivity are helpful in IBS. As few as 5 sessions are 
needed $^{33}$. However, the treatment methods tested in this group of studies have been so varied that they could be considered different forms of treatment. Only progressive relaxation has been tested in more than one of the positive trials. This is a form of physiological relaxation achieved by systematically alternating tensing and relaxing muscles in different muscle groups of the body, and noticing the contrast between the feeling of tension and relaxation. Over time, patients learn in this way to more effectively release muscle tension and relax their body more thoroughly.

\section{Psychodynamic therapy}

Psychodynamic therapy and interpersonal therapy (a less common variant of psychodynamic therapy focused on interpersonal relationships) are insight-oriented therapies that aim at reducing symptoms through gaining an understanding of unconscious processes that may be responsible for those symptoms. Both psychodynamic therapy and interpersonal therapy were reported to improve IBS symptoms in early randomized controlled trials (supplemental Table 4), but the largest and most rigorous RCT of interpersonal therapy for IBS, by Creed and colleagues ${ }^{34}$ failed to show any advantage over standard medical care. Based on this mixed experience and the lack of therapists offering interpersonal therapy, the only type of psychodynamic therapy tested in multiple RCTs for FGIDs, psychodynamic treatment is not considered a generally useful option for FGID patients.

\section{Biofeedback}

Biofeedback is a form of behavioral training that uses continuous visual or auditory feedback from recordings of specific physiological activity to enable patients to learn to voluntarily control those body functions. For example, in a patient who is constipated because she paradoxically contracts her pelvic floor muscles when having a bowel movement, the electromyographic (EMG) activity of her pelvic floor muscles might be shown as a dynamic graph on a computer screen while she simulates defecation to help teach her how to relax the pelvic floor muscles instead of contracting them. The therapist would provide verbal instructions and encouragement during her attempts to relax the muscles. In a patient with fecal incontinence, on the other hand, biofeedback might be used to teach patients how to more effectively contract an external anal sphincter that is very weak due to an obstetrical injury or other causes; in this case biofeedback would be used to teach the patients an appropriate pelvic floor muscle exercise to practice at home to gradually increase the strength of the muscle. Biofeedback can also be used for sensory training, i.e., to improve the patient's ability to detect and respond appropriately to physiological sensations such as stool or gas suddenly filling up the rectum. This type of training would be used in a patient who is unable to recognize when it is necessary to contract the pelvic floor muscles to prevent leaking gas or liquid stool because of a nerve injury. Usually 4-6 training sessions spaced 1-2 weeks apart are used whether the indication is constipation or fecal incontinence.

Pelvic floor biofeedback as described above is distinctly different from the other forms of psychological treatment for FGIDs discussed above because it is not used to cause changes in thoughts or feelings; rather it is utilized to help patients learn to overcome specific physiological deficits directly. A different type of biofeedback is sometimes used to teach patients how to relax all the muscles of their body or to reduce autonomic arousal to counteract stress, but those techniques are very different and rarely used to treat gastrointestinal disorders.

Biofeedback has been tested in randomized studies (supplemental Table 3) almost exclusively as a therapy for functional constipation (16 RCTs) and fecal incontinence (9 RCTs). Comparison conditions have varied, including medical management, sham or non- 
anorectal biofeedback, balloon defecation training, polyethylene glycol, behavioral modification, diazepam, placebo, botox and surgery. Six of 9 RCTs in constipated adults found biofeedback patients to have significantly better outcomes than control patients (Table 5). Of the three negative studies, one was methodologically flawed as it did not limit enrollment to patients with evidence of pelvic floor dyssynergia ${ }^{35}$; it is now evident that biofeedback does not work for constipation unless patients have dyssynergic defecation ${ }^{36}$. The other two negative trials came from a single group of researchers in Egypt ${ }^{37,38}$ who found biofeedback to be less effective compared to botox injection and, in one of the studies, compared to puborectalis surgery as well. In short, it seems that biofeedback is effective in adults with dyssynergic functional constipation, although it may possibly not be as effective as botox injection or surgery. The largest and methodologically strongest trials all show a clinically significant improvement in constipation that is substantially greater in patients treated with biofeedback than in control patients $(70-86 \% \text { vs. } 22-48 \%)^{39-41}$, and therapeutic gains are well maintained for at least 1 year after treatment.

In contrast to the predominantly positive studies in adults, only two of 7 published controlled trials testing biofeedback for constipation in children found an advantage for biofeedback compared to the control conditions. This unfavorable outcome picture may be in part due to two of the trials not limiting testing to dyssynergic patients. It has also been suggested that children may lack the ability to concentrate on the biofeedback task for sustained period of training ${ }^{42}$. In any case, based on the available evidence biofeedback cannot be recommended for treating constipation in children.

Biofeedback has been advocated for the treatment of fecal incontinence since the first case series was described in $1974^{43}$. However, as shown in supplemental Table 5, only 3 of 9 RCTs show superior outcomes of biofeedback treatment compared to control conditions. Both of the 2 trials of biofeedback for encopresis in children yielded negative results ${ }^{44,45}$. The three studies in adults that compared biofeedback to conservative management which combined pelvic floor exercises with education ${ }^{46-48}$ likewise found no advantage for biofeedback, which suggests that biofeedback may not generally help fecal incontinence patients more than those more widely available interventions for fecal incontinence. Much better results were found for biofeedback in adults in a study by Heymen and colleagues ${ }^{49}$, who only enrolled non-responders to a run-in education and medical management intervention in a randomized trial of either biofeedback or pelvic floor exercises alone. Biofeedback showed clear superiority under these circumstances (77\% of patients reported adequate relief versus $48 \%$ in the pelvic floor exercise group), and improvements were well maintained for up to 12 months. These findings suggest that biofeedback is likely to be useful for improving outcomes for adult fecal incontinence patients who are have not had satisfactory response to conservative management.

Biofeedback has also been tested for anorectal pain and functional dyspepsia in single RCTs. In a large trial on 157 individuals with chronic idiopathic anorectal pain ${ }^{50}$, biofeedback resulted in much higher rates of pain relief $(87 \%)$ than were seen for electrogalvanic stimulation (45\%) and levator muscle massage (22\%) conditions, and treatment benefits were maintained at one-year follow-up. Thus, biofeedback holds strong promise for the future as a possible advancement in the management of this difficult-to-treat problem. The only trial of biofeedback for functional dyspepsia ${ }^{51}$ did not report the effect of this treatment on dyspepsia symptoms (but reported biofeedback to result in greater drinking capacity and quality of life improvement compared to no treatment) and therefore provided little direct information about the potential value of this therapy for improving the clinical symptoms of dyspepsia. 
As the above summary of these five psychological treatments illustrates, a vast amount of published RCT data support the value of psychological therapies as interventions for FGIDs. It should be acknowledged that many of the trials in this domain have methodological shortcomings: The great majority of them have been small; many have used waiting lists or mere symptom monitoring as control groups, both of which are likely to produce negative expectation of improvement that may exaggerate outcome contrast with the active treatment. Therapies are sometimes poorly described, making it hard to know exactly what therapy was tested or how to replicate it. Results have also been measured in numerous different ways that make it hard to compare outcomes across trials. Nonetheless, the preponderance of evidence, buttressed by some methodologically strong trials for each psychological modality tested, makes a strong case for the psychological treatment as adjunctive therapy options to consider for FGID patients.

\section{Which patients should receive psychological treatment?}

Psychological treatment is neither necessary nor reasonable for most FGID patients. A substantial proportion, especially those with relatively mild symptoms, gain sufficient symptom relief from the usual medical care provided in gastroenterology and primary care practices. The extra healthcare costs and substantial time and effort that psychological interventions require may not be warranted for those individuals. However, psychological treatment should in our opinion generally be considered for two types of patients:

1. Patients who continue to have moderate or severe symptoms after 3 to 6 months of medical management

2. Patients whose case presentation suggests that stress or emotional symptoms are likely to be exacerbating GI symptoms or impairing coping with illness.

Not all patients within these two categories are equally well suited for referral. Individuals who do not recognize or are unwilling to accept the role that stress or psychological symptoms influence the severity of their gastrointestinal symptoms will not be likely to follow through with such treatment. Patients with disabling psychiatric symptoms or thought disorder may have difficulty complying with a psychological treatment regimen for FGIDs, and could require treatment with psychotropic medications prescribed by a psychiatrist. Patients who are very unmotivated to assume an active role in managing their own health condition may also do poorly with psychological treatment, which requires considerable effort and work over a number of sessions with a therapist.

\section{Choosing appropriate psychological treatment}

What kind of psychological treatment is selected for each patient will be influenced to some degree by local availability of the different psychological services, patient preference, and the clinician's past experience with referrals for the problems to be addressed. However, the following general guidelines can be given about the choices that are most likely to yield good results, based on the literature to date:

a. Psychological treatment to improve gastrointestinal symptoms. If the goal of the referral is to achieve better improvement in GI symptoms than has been possible with medical management, cognitive-behavioral therapy and hypnosis offer excellent chances of improvement in IBS symptoms and pediatric abdominal pain. Relaxation training (especially progressive muscle relaxation) is also a suitable option for IBS treatment. Non-cardiac chest pain shows a good response to CBT. For constipation due to pelvic floor dyssynergia in adults, biofeedback is a good option. However, slow transit constipation without evidence of dyssynergic defecation is unresponsive to this therapy. Functional abdominal pain in children 
can be treated effectively with family CBT and hypnosis. There is insufficient empirical evidence to recommend particular therapies for other FGIDs, but single sizable and methodologically strong RCTs suggest that biofeedback might be worth consideration for levator ani syndrome (especially if patients have puborectalis tenderness on physical examination) ${ }^{50}$ and hypnosis for functional dyspepsia ${ }^{31}$.

b. Psychological treatment for co-morbid affective symptoms. If the primary goal of referral is reduction in co-morbid emotional symptoms such as anxiety or depression, cognitive-behavioral therapy is generally the best choice, as there is somewhat better evidence than for other forms of psychotherapy that CBT can reliably reduce these affective symptoms in a short course of treatment ${ }^{52,53}$. CBT is also particularly well suited for helping patients with maladaptive coping styles, such as a tendency to catastrophize or excessive pre-occupation with bowel symptoms, which can impair life functioning and quality of life in FGIDs. If life stress or stress-related symptoms is thought to be a key problem and the patient is able to recognize this, relaxation therapies are likely to be well received and effective. If somatization, i.e. the psychological tendency to experience a multitude of non-specific body symptoms is a prominent problem, hypnosis treatment may be a suitable treatment option since it has been more often shown to reduce nongastrointestinal symptoms in FGIDs than other therapies ${ }^{23,}$, $4,55$.

\section{How to best ensure effective referral for psychological treatment}

Referral of a FGID patient for psychological treatment is often a delicate matter. Patients generally consult a gastroenterologist with the expectation of being investigated and treated for organic disease. They may be unaware of the influences of emotions and the brain on gastrointestinal functioning and may not see psychological treatment as relevant to their GI problems. Any suggestion of such treatment is easily misunderstood as indicating that their gut symptoms are a mere psychiatric problem. For good probability of success, psychological referral therefore requires tact, correct timing, good doctor-patient communication, ensuring that the patient clearly understands the rationale for referral, and also considerable effort on the part of the doctor or clinic staff to manage the referral. The following steps can help to ensure high probability of successful referral:

a. Introduce the role of psychological influences and psychological treatment early with FGID patients. When a psychological referral is mentioned for the first time after a course of medical treatment has failed to produce satisfactory results, the patient is more likely to interpret this as the physician giving up on finding the cause or treatment for the gastrointestinal symptoms. Conversely, if psychological treatment has been a part of the picture the patient has of management of the disorder from the beginning, it may instead seem like the logical next step in treatment efforts. This early introduction of the brain-gut relationship and psychological treatment in the disorder can be in the form of a brochure given at the end of the first visit, as well as incorporated in education of the patient when the diagnosis is verbally explained.

b. Reassure the patient that the correct diagnosis has been made. Patients who think that they may have an organic disease and have little confidence in their functional GI diagnosis are unlikely to be accepting of referral for psychological therapy, as they are likely to see it as inappropriate and even a reckless distraction from pursuing the "real" cause of their symptoms. Thorough explanation of the disorder and the reasons for confidence in the diagnosis, coupled with reassurance that it is highly unlikely that alternative dangerous medical problems can account for the symptoms, is advisable before referral for psychological treatment is discussed. 
c. Establish a firm therapeutic alliance. If the physician makes clear that he or she is committed to working with the patient to pursue whatever means are possible to achieve the best symptom reduction and quality-of-life enhancement, and discusses the different options for this purpose with the patient as a partner in that endeavor, this sets the stage for discussing psychological treatment as a logical part of overall symptom management.

d. Explain thoroughly the rationale for the psychological treatment. As emphasized by Drossman and colleagues in the AGA technical review on $\mathrm{IBS}^{3}$, explaining to FGID patients the rationale for referral for psychological treatment is crucial. It may be the single-most important factor for a referral to work. This explanation should first of all include why psychological treatment is likely to help. For that purpose, explanation of the brain-gut axis and the way the brain down-regulates or amplifies pain perception and gut activity is essential. One should also explain that this control is tuned by both strong emotions and stress, and that psychological treatment can use that same mind-body relationship to neutralize symptoms and the impact of emotions. As the effects of stress and the gut is apparent to the patient from his or her own experience, examples such as nausea, butterflies in the stomach or lump in the throat in response to strong emotions can help illustrate this discussion. Secondly, the fact that numerous studies show that psychological treatments improve outcomes for patients with FGID compared to medical treatment alone should be discussed, and especially the evidence for the particular therapy being proposed.

e. Identify suitable local service providers. There is great advantage to identifying one or more suitable therapists for referral in the community, and learning which therapies they can provide that are appropriate for FGIDs, before referral is discussed with the patient. Relying on the patients' initiative to find an appropriate therapist is unlikely to succeed, as their understanding of what is required may be limited. Taking the trouble to find suitable local therapists and learn about their services can pay off handsomely, since a good provider can be utilized again and again for referrals. If the gastroenterologist already knows what type of psychological treatment will be used and can describe how it works in general terms, this is more credible to the patient and can facilitate follow-through. Appropriate therapists are generally clinicians who are experienced in treating physical health problems, and preferably gastrointestinal disorders, with psychological methods. In general, mental health providers who list health psychology or behavioral medicine as their focus of practice are likely candidates for referral. For finding therapists who provide particular types of psychological services most suitable for FGIDs, consulting online resources for therapist referrals can also be helpful. Useful websites for finding therapists in any part of the U.S. include www.abct.org and academyofct.org for CBT, www.asch.net and www.ibshypnosis.com for hypnosis, and www.bcia.org for pelvic floor biofeedback. Physicians practicing in small towns or rural areas may not be able to find providers of these psychological services locally, but should consider locating providers in the nearest major urban center, as patients may be willing to travel some distance for a short course of such specialized treatment.

f. Communicate with the psychological services provider. Many GI patients will not be able to effectively convey the goals of referral for their FGID problem to the psychological services provider. It can therefore be greatly advantageous to provide the patient with a referral letter explaining the rationale and expectations for the referral. The letter should make clear that what is being sought is a brief course of adjunctive therapy and it should be clearly stated whether the desired principal goal 
of the referral is to treat affective symptoms or the GI symptoms. It can also be very helpful to request a written report back from the therapist if treatment does not seem appropriate or if therapy response is poor, so that other options can be selected instead, and to encourage the provider to telephone about coordinating psychological treatment with ongoing medical treatment.

g. Place emphasis on continuation of GI care. Emphasizing both to the patient at the time of referral and in the referral letter to the therapist that the gastroenterologist will continue to manage the overall GI care of the patient can reassure patients who have such anxieties that he or she is not being "dumped" and give the therapist a better understanding of the context for his or her work with the patient. Encouraging the patient to schedule a return GI visit at the end of the course of psychological treatment to assess progress and decide on next steps if needed will further reinforce this sense of continuation of care and strengthen the doctor-patient therapeutic alliance.

\section{Psychopharmacologic treatment as an alternative to psychological treatment}

Psychotropic medications, and especially anti-depressant, have been shown to have utility in the treatment of FGIDs in a number of studies. These medications can sometimes be used to a significant degree to accomplish the two main tasks that we have described for FGID psychotherapy in this article: To reduce GI symptom intensity and manage co-morbid affective symptoms ${ }^{56}$. Use of antidepressants has become fairly well established for functional GI symptoms, especially in IBS. At least one in every eight IBS patients is offered antidepressant medication ${ }^{57}$. Ford and colleagues recently conducted a systematic review of both RCTs of antidepressant medication and psychological therapies in IBS, and concluded that the number needed to treat (NNT) was 4 for both types of intervention ${ }^{58}$. Psychotropic medications may therefore be a suitable alternative to psychological treatment for many patients, and this option has the pronounced advantage of not requiring outside referral. However, psychotropic drugs also have some limitations relative to psychological treatments. The medications that have the best evidence of effectiveness in therapeutic value for FGID symptoms are old-style tricyclic anti-depressants that have relatively unfavorable side effect profile 56 . Some of the most common side effects of antidepressants in general are gastrointestinal symptoms such as nausea and constipation, and the safety of these medications in patients with gastrointestinal disorders is not well known ${ }^{2}$. Furthermore, psychotropic medications can interact with other drugs the patients are taking. Finally, unlike the effects of psychological treatments, which often last many months or even years beyond the end of treatment, psychotropic medications need to be administered chronically for lasting benefit.

\section{Summary and conclusions: Advantages and limitations of psychological treatment}

As our summary of individual treatments above demonstrates, a substantial literature supports the value of psychological treatment for patients with FGIDs. Good improvement is often seen from such treatment in patients who have shown little or no response to usual medical care. These therapies have no adverse side effects and do not cause interactions with pharmacologic treatment, making them well suited as co-therapies with medical care. Apart from improvement in symptoms these therapies often bring patients positive outcomes including better quality of life, enhanced emotional well-being and life functioning, improved coping with the illness, and lessened healthcare and medication needs. The 
prospects of such improved well-being and functional outcomes are sufficient benefits to warrant referrals for these therapies for some patients.

There are limitations and challenges to the utilization of psychological treatments in the FGIDs, however. For good success, referrals for such treatment require good therapeutic alliance between doctor and patient, reassurance and education. It may be hard to find therapists in some areas who are skilled in the particular therapy modalities with best evidence of effectiveness for particular FGIDs. Also, the amount of effort and motivation required of patients to make use of psychological treatments may make it unsuitable for some individuals. Insurance reimbursement for psychological therapies for FGIDs is variable and not available in all insurance plans, so cost may be an obstacle for some patients.

In addition to these limitations that are common to all the treatment modes, the various treatments also have different challenges. The idea of hypnosis treatment may cause wariness in some patients due to the common misconceptions about hypnosis in mass media and the general culture. Cognitive behavioral therapy generally depends heavily on homework assignments and therefore requires diligent and self-motivated patients for the best results. Biofeedback is more invasive than the other forms of psychological treatment, and some patients may be uncomfortable with use of intra-rectal sensors. Finally, the research to date has only identified effective psychological treatments for a few of the many FGIDs. Despite all of these limitations, the current state of knowledge clearly indicates that psychological treatment should be considered for the subgroup of FGID patients who do not gain satisfactory symptom relief from usual gastroenterology management, and is likely to significantly improve their clinical outcomes.

\section{Supplementary Material}

Refer to Web version on PubMed Central for supplementary material.

\section{Acknowledgments}

Supported by grants R01 DK031369 from the NIDDK And grant R01 HS018695 from the Agency for Healthcare Research and Quality

\section{References}

1. Whitehead WE, Levy RL, Von Korff M, et al. The usual medical care for irritable bowel syndrome. Aliment Pharmacol Ther. 2004; 20:1305-15. [PubMed: 15606392]

2. Brandt LJ, Chey WD, Foxx-Orenstein AE, et al. An evidence-based position statement on the management of irritable bowel syndrome. Am J Gastroenterol. 2009; 104 (Suppl 1):S1-35. [PubMed: 19521341]

3. Drossman DA, Camilleri M, Mayer EA, et al. AGA technical review on irritable bowel syndrome. Gastroenterology. 2002; 123:2108-31. [PubMed: 12454866]

4. Whitehead WE. Psychosocial aspects of functional gastrointestinal disorders. Gastroenterol Clin North Am. 1996; 25:21-34. [PubMed: 8682574]

5. Chitkara DK, van Tilburg MA, Blois-Martin N, et al. Early life risk factors that contribute to irritable bowel syndrome in adults: a systematic review. Am J Gastroenterol. 2008; 103:765-74. quiz 775. [PubMed: 18177446]

6. Whitehead WE, Palsson O, Jones KR. Systematic review of the comorbidity of irritable bowel syndrome with other disorders: what are the causes and implications? Gastroenterology. 2002; 122:1140-56. [PubMed: 11910364]

7. Drossman DA. Do psychosocial factors define symptom severity and patient status in irritable bowel syndrome? Am J Med. 1999; 107:41S-50S. [PubMed: 10588172] 
8. Van Oudenhove L, Vandenberghe J, Vos R, et al. Risk factors for impaired health-related quality of life in functional dyspepsia. Aliment Pharmacol Ther. 2011; 33:261-74. [PubMed: 21083672]

9. Levy RL, Olden KW, Naliboff BD, et al. Psychosocial aspects of the functional gastrointestinal disorders. Gastroenterology. 2006; 130:1447-58. [PubMed: 16678558]

10. Van Oudenhove L, Vandenberghe J, Geeraerts B, et al. Relationship between anxiety and gastric sensorimotor function in functional dyspepsia. Psychosom Med. 2007; 69:455-63. [PubMed: 17556644]

11. Clauwaert N, Jones MP, Holvoet L, et al. Associations between gastric sensorimotor function, depression, somatization, and symptom-based subgroups in functional gastroduodenal disorders: are all symptoms equal? Neurogastroenterol Motil. 2012

12. Posserud I, Agerforz P, Ekman R, et al. Altered visceral perceptual and neuroendocrine response in patients with irritable bowel syndrome during mental stress. Gut. 2004; 53:1102-8. [PubMed: 15247175]

13. Payne A, Blanchard EB. A controlled comparison of cognitive therapy and self-help support groups in the treatment of irritable bowel syndrome. J Consult Clin Psychol. 1995; 63:779-86. [PubMed: 7593870]

14. Moss-Morris R, McAlpine L, Didsbury LP, et al. A randomized controlled trial of a cognitive behavioural therapy-based self-management intervention for irritable bowel syndrome in primary care. Psychol Med. 2010; 40:85-94. [PubMed: 19531276]

15. Craske MG, Wolitzky-Taylor KB, Labus J, et al. A cognitive-behavioral treatment for irritable bowel syndrome using interoceptive exposure to visceral sensations. Behav Res Ther. 2011; 49:413-21. [PubMed: 21565328]

16. van Dulmen AM, Fennis JF, Bleijenberg G. Cognitive-behavioral group therapy for irritable bowel syndrome: effects and long-term follow-up. Psychosom Med. 1996; 58:508-14. [PubMed: 8902902]

17. Klimes I, Mayou RA, Pearce MJ, et al. Psychological treatment for atypical non-cardiac chest pain: a controlled evaluation. Psychol Med. 1990; 20:605-11. [PubMed: 2236369]

18. Mayou RA, Bryant BM, Sanders D, et al. A controlled trial of cognitive behavioural therapy for non-cardiac chest pain. Psychol Med. 1997; 27:1021-31. [PubMed: 9300508]

19. Van Peski-Oosterbaan AS, Spinhoven P, Van der Does AJ, et al. Cognitive change following cognitive behavioural therapy for non-cardiac chest pain. Psychother Psychosom. 1999; 68:21420. [PubMed: 10396013]

20. Spinhoven P, Van der Does AJW, Van Dijk E, et al. Heart-focused anxiety as a mediating variable in the treatment of noncardiac chest pain by cognitive-behavioral therapy and paroxetine. $\mathrm{J}$ Psychosom Res. 2010; 69:227-235. [PubMed: 20708444]

21. Jonsbu E, Dammen T, Morken G, et al. Short-term cognitive behavioral therapy for non-cardiac chest pain and benign palpitations: a randomized controlled trial. J Psychosom Res. 2011; 70:11723. [PubMed: 21262413]

22. National Institute of Diabetes and Digestive and Kidney Diseases (NIDDK), State University of New York at Buffalo. ClinicalTrials.gov [Internet]. Bethesda (MD): National Library of Medicine (US); 2000. Self Administered Cognitive Behavior Therapy for Irritable Bowel Syndrome. [cited 2012 Oct 8]. Available from: http://clinicaltrials.gov/ct2/show/NCT00738920 NLM Identifier: NCT00738920

23. Palsson OS, Turner MJ, Johnson DA, et al. Hypnosis treatment for severe irritable bowel syndrome: investigation of mechanism and effects on symptoms. Dig Dis Sci. 2002; 47:2605-14. [PubMed: 12452403]

24. Whorwell PJ, Prior A, Colgan SM. Hypnotherapy in severe irritable bowel syndrome: further experience. Gut. 1987; 28:423-5. [PubMed: 3583070]

25. Gonsalkorale WM, Miller V, Afzal A, et al. Long term benefits of hypnotherapy for irritable bowel syndrome. Gut. 2003; 52:1623-9. [PubMed: 14570733]

26. Vlieger AM, Menko-Frankenhuis C, Wolfkamp SC, et al. Hypnotherapy for children with functional abdominal pain or irritable bowel syndrome: a randomized controlled trial. Gastroenterology. 2007; 133:1430-6. [PubMed: 17919634] 
27. Vlieger AM, Rutten JM, Govers AM, et al. Long-term follow-up of gut-directed hypnotherapy vs. standard care in children with functional abdominal pain or irritable bowel syndrome. Am J Gastroenterol. 2012; 107:627-31. [PubMed: 22310221]

28. van Tilburg MA, Chitkara DK, Palsson OS, et al. Audio-recorded guided imagery treatment reduces functional abdominal pain in children: a pilot study. Pediatrics. 2009; 124:e890-7. [PubMed: 19822590]

29. Jones H, Cooper P, Miller V, et al. Treatment of non-cardiac chest pain: a controlled trial of hypnotherapy. Gut. 2006; 55:1403-8. [PubMed: 16627548]

30. Miller V, Jones H, Whorwell PJ. Hypnotherapy for non-cardiac chest pain: long-term follow-up. Gut. 2007; 56:1643. [PubMed: 17938446]

31. Calvert EL, Houghton LA, Cooper P, et al. Long-term improvement in functional dyspepsia using hypnotherapy. Gastroenterology. 2002; 123:1778-85. [PubMed: 12454833]

32. Boyce PM, Talley NJ, Balaam B, et al. A randomized controlled trial of cognitive behavior therapy, relaxation training, and routine clinical care for the irritable bowel syndrome. Am $\mathrm{J}$ Gastroenterol. 2003; 98:2209-18. [PubMed: 14572570]

33. van der Veek PP, van Rood YR, Masclee AA. Clinical trial: short- and long-term benefit of relaxation training for irritable bowel syndrome. Aliment Pharmacol Ther. 2007; 26:943-52. [PubMed: 17767479]

34. Creed F, Fernandes L, Guthrie E, et al. The cost-effectiveness of psychotherapy and paroxetine for severe irritable bowel syndrome. Gastroenterology. 2003; 124:303-17. [PubMed: 12557136]

35. Koutsomanis D, Lennard-Jones JE, Roy AJ, et al. Controlled randomised trial of visual biofeedback versus muscle training without a visual display for intractable constipation. Gut. 1995; 37:95-9. [PubMed: 7672690]

36. Chiarioni G, Salandini L, Whitehead WE. Biofeedback benefits only patients with outlet dysfunction, not patients with isolated slow transit constipation. Gastroenterology. 2005; 129:8697. [PubMed: 16012938]

37. Farid M, El Monem HA, Omar W, et al. Comparative study between biofeedback retraining and botulinum neurotoxin in the treatment of anismus patients. Int J Colorectal Dis. 2009; 24:115-20. [PubMed: 18719924]

38. Faried M, El Nakeeb A, Youssef M, et al. Comparative study between surgical and non-surgical treatment of anismus in patients with symptoms of obstructed defecation: a prospective randomized study. J Gastrointest Surg. 2010; 14:1235-43. [PubMed: 20499203]

39. Rao SS, Seaton K, Miller M, et al. Randomized controlled trial of biofeedback, sham feedback, and standard therapy for dyssynergic defecation. Clin Gastroenterol Hepatol. 2007; 5:331-8. [PubMed: 17368232]

40. Heymen S, Scarlett Y, Jones K, et al. Randomized, controlled trial shows biofeedback to be superior to alternative treatments for patients with pelvic floor dyssynergia-type constipation. Dis Colon Rectum. 2007; 50:428-41. [PubMed: 17294322]

41. Chiarioni G, Whitehead WE, Pezza V, et al. Biofeedback is superior to laxatives for normal transit constipation due to pelvic floor dyssynergia. Gastroenterology. 2006; 130:657-64. [PubMed: 16530506]

42. Chiarioni G, Whitehead WE. The role of biofeedback in the treatment of gastrointestinal disorders. Nat Clin Pract Gastroenterol Hepatol. 2008; 5:371-82. [PubMed: 18521115]

43. Engel BT, Nikoomanesh P, Schuster MM. Operant conditioning of rectosphincteric responses in the treatment of fecal incontinence. N Engl J Med. 1974; 290:646-9. [PubMed: 4813725]

44. Wald A, Chandra R, Gabel S, et al. Evaluation of biofeedback in childhood encopresis. J Pediatr Gastroenterol Nutr. 1987; 6:554-8. [PubMed: 3430263]

45. van der Plas RN, Benninga MA, Redekop WK, et al. Randomised trial of biofeedback training for encopresis. Arch Dis Child. 1996; 75:367-74. [PubMed: 8957948]

46. Ilnyckyj A, Fachnie E, Tougas G. A randomized-controlled trial comparing an educational intervention alone vs education and biofeedback in the management of faecal incontinence in women. Neurogastroenterol Motil. 2005; 17:58-63. [PubMed: 15670265]

47. Norton C, Chelvanayagam S, Wilson-Barnett J, et al. Randomized controlled trial of biofeedback for fecal incontinence. Gastroenterology. 2003; 125:1320-9. [PubMed: 14598248] 
48. Solomon MJ, Pager CK, Rex J, et al. Randomized, controlled trial of biofeedback with anal manometry, transanal ultrasound, or pelvic floor retraining with digital guidance alone in the treatment of mild to moderate fecal incontinence. Dis Colon Rectum. 2003; 46:703-10. [PubMed: 12794569]

49. Heymen S, Scarlett Y, Jones K, et al. Randomized controlled trial shows biofeedback to be superior to pelvic floor exercises for fecal incontinence. Dis Colon Rectum. 2009; 52:1730-7. [PubMed: 19966605]

50. Chiarioni G, Nardo A, Vantini I, et al. Biofeedback is superior to electrogalvanic stimulation and massage for treatment of levator ani syndrome. Gastroenterology. 2010; 138:1321-9. [PubMed: 20044997]

51. Hjelland IE, Svebak S, Berstad A, et al. Breathing exercises with vagal biofeedback may benefit patients with functional dyspepsia. Scand J Gastroenterol. 2007; 42:1054-62. [PubMed: 17710670]

52. Nieuwsma JA, Trivedi RB, McDuffie J, et al. Brief psychotherapy for depression: a systematic review and meta-analysis. Int J Psychiatry Med. 2012; 43:129-51. [PubMed: 22849036]

53. Hofmann SG, Smits JA. Cognitive-behavioral therapy for adult anxiety disorders: a meta-analysis of randomized placebo-controlled trials. J Clin Psychiatry. 2008; 69:621-32. [PubMed: 18363421]

54. Gonsalkorale WM, Houghton LA, Whorwell PJ. Hypnotherapy in irritable bowel syndrome: a large-scale audit of a clinical service with examination of factors influencing responsiveness. Am J Gastroenterol. 2002; 97:954-61. [PubMed: 12003432]

55. Houghton LA, Heyman DJ, Whorwell PJ. Symptomatology, quality of life and economic features of irritable bowel syndrome--the effect of hypnotherapy. Aliment Pharmacol Ther. 1996; 10:91-5. [PubMed: 8871448]

56. Grover M, Drossman DA. Psychotropic agents in functional gastrointestinal disorders. Curr Opin Pharmacol. 2008; 8:715-23. [PubMed: 18760380]

57. Clouse RE, Lustman PJ. Use of psychopharmacological agents for functional gastrointestinal disorders. Gut. 2005; 54:1332-41. [PubMed: 16099800]

58. Ford AC, Talley NJ, Schoenfeld PS, et al. Efficacy of antidepressants and psychological therapies in irritable bowel syndrome: systematic review and meta-analysis. Gut. 2009; 58:367-78. [PubMed: 19001059] 